Article

\title{
Facebook Users' Engagement and Perceived Life Satisfaction
}

\author{
Tammy R. Vigil * and H. Denis Wu \\ College of Communication, Boston University, Boston, MA 02215, USA; E-Mails: tvigil@bu.edu (T.R.V.), \\ hdw@bu.edu (H.D.W.) \\ * Corresponding author
}

Submitted: 17 October 2014 | In Revised Form: 29 April 2015 | Accepted: 22 May 2015 |

Published: 20 July 2015

\begin{abstract}
This study extends existing research on Facebook's impact on users' life satisfaction. The results from two surveys of college students demonstrate a tension between Facebook use and users' perceived contentment with their lives. Existing literature indicates students use Facebook to enhance self-esteem, yet the results from this study connect increased Facebook use to lower self-reported levels of happiness. In particular, respondents' interactions with photos and videos increase users' dissatisfaction. This phenomenon may be due to the impact photos have on the ways users engage in social comparisons with Facebook "friends" and the self-construals they create based on these comparisons.
\end{abstract}

\section{Keywords}

Facebook; happiness; life satisfaction; self-construal; social comparison; social media

\section{Issue}

This article is part of a regular issue of Media and Communication, edited by Professor Bradley Greenberg (Michigan State University, USA) and Professor Elisabeth Klaus (University of Salzburg, Austria).

(C) 2015 by the authors; licensee Cogitatio (Lisbon, Portugal). This article is licensed under a Creative Commons Attribution 4.0 International License (CC BY).

\section{Introduction}

Over recent years, the number of studies examining various facets of the Internet and its impacts has continually increased. Much of that growing research focuses on social networking and information sharing. Existing scholarship about online communication ranges from descriptive examinations of use (Attrill \& Jalil, 2011; Hwang, 2011; Quan-Haase \& Young, 2010; Valkenburg \& Peter, 2011) to specific content analysis of participant contributions (Ellison, Steinfield, \& Lampe, 2011; Nosko, Wood, \& Molema, 2010) and experimental tests of influence (Antheunis, Valkenburg, \& Peter, 2010; Kross et al., 2013).

While much of the existing research regarding online communication looks generally at different types of content and the impact of various online functions, there is particular interest in the specific impact and influence of Facebook, a widely used social networking application (Cheung, Chiu, \& Lee, 2011). Facebook has attracted several hundred million users worldwide (Who Uses Facebook, 2012) and young people comprise the largest percentage of participants in the Facebook world (Engagement Analytics, 2012). Studies focusing on Facebook as a social medium include examinations of website content (Hum et al., 2011), the types and amounts of self-disclosure users provide (Nosko et al., 2010), motivations for using Facebook (Baek, Holton, Harp, \& Yaschur, 2011; Cheung et al., 2011; Nadkarni \& Hofmann, 2011; Pempek, Yermolayeva, \& Colvert, 2009; Quan-Haase \& Young, 2010; Tosun, 2012) and the impact of personality on Facebook use (Muscanell \& Guadango, 2012; Nadkarni \& Hofnann, 2011; Ryan \& Xenos, 2011; Sukes, Williams, \& Wise, 2012). In addition, scholars have created typologies of media uses and gratifications emphasizing social networking (Bumgarner, 2007; Cheung et al., 2011; Quan-Hass \& Young, 2010; Sheldon, Abad, \& Hinsch, 2011; Smock, Ellison, Lampe, \& Whon, 2011). Some studies show that Facebook use is driven by a variety of different functions (Pempek et al., 2009; Smock et al., 2011) and can challenge the traditional demarcation of 
motivations for media use (Sheldon et al., 2011).

This study uses the theories of social comparison and self-construal to untangle the effects of Facebook use on users' reported life satisfaction. We examine the utility of these two theories in explicating the impact of mediated interpersonal communication on how individuals perceive their lives and the positive or negative judgments they make about those perceptions. Two online surveys of undergraduate students at a large university in the Northeast provide a wealth of information regarding respondents' use of the social networking medium. Consistent with expectations based on the social process of creating self-construals, the surveys investigated sex-based differences in Facebook use habits and preferences, many of which correlate with alterations in perceived levels of life satisfaction. The surveys also explored how variation in the ways respondents interact with Facebook is related to users' reported satisfaction with their own lives, a phenomenon that may be better understood through the models of upward and downward social comparison to others. The overall purpose of this study is to expand understanding of how Facebook use influences life satisfaction based on the concepts gleaned from "offline" communication theories. To better accomplish this goal, we begin with a review of relevant studies on Facebook and existing literature surrounding social comparisons and self-construal.

\section{Literature Review}

Previous examinations of college-aged individuals' use of Facebook have revealed consistent and similar patterns of use. College students check their established Facebook accounts on a daily basis (Nadkarni \& Hofmann, 2011; Quan-Haase \& Young, 2011; Vitak et al., 2011) and spend between 30-60 minutes per day on the website (Ellison, Seinfield, \& Lempke, 2007; Ellison et al., 2011; Pempek et al., 2009; Quan-Haase \& Young, 2010). Some studies show that individuals often "lurk" online, reading others' information without posting or responding in a reciprocal manner (Bumgarner, 2007; Pempek et al., 2009). This lurking behavior indicates that Facebook users tend to engage in various uncertainty reduction strategies to acquire information about acquaintances and friends (Antheunis et al., 2010) but do not always reciprocate with similar online disclosures. The lurking behavior gratifies some users' need for perceived maintenance of interpersonal connectivity (Cheung et al., 2011; Sheldon et al., 2011) without risking their own social identity by disclosing face-threatening personal information (Arundale, 2010; Nosko et al., 2010) or expressing potentially objectionable opinions that may disrupt affinity-seeking strategies (Hwang, 2011).

Most scholars focusing on Facebook agree that users primarily engage the networking site to maintain contact with individuals with whom they have existing offline relationships (Cheung et al., 2011; Ellison et al., 2011; Muise, Christofides, \& Desmarais, 2009; Nosko et al., 2010; Pempeck et al., 2009; Sheldon et al., 2011). As Quan-Haase and Young (2010) explain, users seek social knowledge from Facebook to feel "involved in what is going on with others" or to be socially "in the know" (p. 355). The desire to be socially knowledgeable about others motivates lurking behavior online. It may also elicit participation via disclosure from those embracing the asynchronous nature of the exchange (Antheunis et al., 2010).

Several studies on the impact of Facebook have shown that participation in online social networking may have positive effects. Ellison et al. (2007) identified a positive relationship between the acquisition of Facebook "friends" and users' perceived growth in social capital, primarily due to the "crystallization" (via Facebook) of otherwise ephemeral social connections. Some scholars argue that such increased social capital favorably impacts individuals' well-being on multiple levels (Ferlander, 2007). A 2011 study by Ellison et al., demonstrates that Facebook-enabled communication practices result in connection-building strategies that can positively impact physical health. Increases in social capital are also linked to mentally healthy behaviors such as increases in self-esteem and enhanced identity formation, particularly in adolescents and young adults (Cheung et al., 2011; Valkenburg \& Peter, 2011). The ability to enhance key elements of one's identity is viewed as positive because "there is a universal desire among human beings to maintain, protect and enhance their self-esteem" (Valkenburg \& Peter, 2011, p. 123).

In contrast to the positive influences found in some studies, others indicate potential negative impacts of Facebook use. Sheldon et al. (2011) argue that Facebook use can heighten users' feeling of social disconnectedness by creating transient perceptions of connectedness. Chou and Edge (2012) conclude that Facebook gives users the impression that their Facebook friends have better lives than they do, thereby encouraging negative self-assessments. They report a decrease in overall life satisfaction and a lower reported level of happiness by frequent Facebook users. This finding depends largely on the closeness of the relationship between the user and his or her Facebook "friends" (Chou \& Edge, 2012). In addition, Kross and his colleagues (2013) demonstrate that increased Facebook use contributes to declines in users' perceived "moment-to-moment" feelings of well-being and their satisfaction with their lives. This particular aspect of social media effect is intriguing and merits further investigation. One of the potential explanations to this phenomenon can be social comparison theories.

\subsection{Social Comparisons}

Social comparison theories argue that humans tend to 
assess their own social standing, attributes, skills and other self-defining elements relative to others about whom they have access to information (Wheeler \& Miyake, 1992). These points of access may be workrelated (Fischer, Kastenmuller, Frey, \& Peus, 2009), personal or romantic (Brewer \& Weber, 1994; Salovey \& Rodin, 1984), or mediated (Wilcox \& Laird, 2000). The information acquired may be partial or complete, verbal or nonverbal, verified or invalid, and still have an impact on the social comparison (Brewer \& Weber, 1994; Salovey \& Rodin, 1984; Wilcox \& Laird, 2000). The act of comparing the self to others can result in self-stereotyping and may encourage shifting selfperceptions to focus on group identity norms rather than individual norms and characteristics (Attrill \& Jalil, 2011). Because Facebook is a medium for social exchange, an essential characteristic of its use is increased access to information about and from others (Valkenburg \& Peter, 2011). Facebook, by its very purpose, encourages users to engage in social comparison with others.

The types of information sought and the directionality of the comparisons influence the power and outcome of social comparison. These two factors influence each other; the type of information that one accesses about another influences the directionality of social comparison, which, in turn, affects its valence. Lifestyle information (i.e., how one lives, activities engaged in, etc.) is primarily sought data requiring a certain degree of intent-driven information seeking. In offline communication, one must pursue this type of information or glean it from other disclosure (e.g., co-workers do not necessarily know about others' home lives unless an inquiry is made) (Fischer et al., 2009). Because individuals have some control over the information sought, exchanges focusing on lifestyle are often associated with downward comparisons (i.e., perceiving one's self as superior). Downward comparisons tend to result in more positive affective reactions (Wheeler \& Miyake, 1992). Asset information is considered forced data because it is presented via the "raw materials" of personal information (i.e., abilities, social skills and appearance). These pieces of information are accessed with little or no intent and are not usually sought out. An individual has little control over the amount of asset information received during any given exchange (Sharkey $\&$ Singelis, 1995). Comparisons made under these circumstances are less deliberate and are more likely to have a negative impact (Wheeler \& Miyake, 1992).

Because of the nature of social media, its influence derived from either type of information accessed is complicated. The act of logging on with the express purpose of checking Facebook content implies a large degree of intent. However, once on the site, the degree of interactive and passive information gathered actually hinges on the online behavior of the user (Smock et al., 2011). Because the tools on Facebook encourage varying levels of activity, users are likely to acquire a mix of asset and lifestyle information and a mix of sought (searching another's profile, engaging in a Facebook messenger conversation, etc.) versus forced (news feed posts, others' photos, etc.) information (Nosko et al., 2010). In addition, the way that information is disclosed on Facebook means lifestyle information is more readily available without particular inquiry than it otherwise might be in offline exchanges (Antoci, Sabatini, \& Sodini, 2012). This is particularly true of posted pictures and videos that readily disclose lifestyle information (Hum et al., 2011). Thus, while the user may intentionally seek specific kinds of information, which does not mean only the sought information is received. This creates ample opportunities for a variety of upward and downward comparisons for Facebook users.

In addition to the mix of types of information received, the relationship between the respondent and the person serving as the point of comparison also influences the directionality of a social comparison (Fischer et al., 2009; Wheeler \& Miyake, 1992). There is a tendency to make same-level comparison with close friends, downward comparisons with "ordinary" friends, and upward comparison with distant friends, acquaintances and strangers (Wheeler \& Miyake, 1992). These findings indicate that social comparisons are rooted in familiarity with and regard for the object of comparison. The better one "knows" the other, the more neutral the comparison. The less well one knows the other, the more he or she relies on the available information for the creation of perceptions about the other. Therefore, perceived closeness of "friends" may be an important factor when using social comparison to hypothesize about Facebook effects.

While traditional social comparison findings are grounded in research outside of mediated interpersonal communication, it provides a potentially rich perspective for understanding Facebook and its influences (Hwang, 2011). Based on social comparison research, the more an individual uses Facebook, the more he or she will socially compare him- or herself to others. Because individuals are less likely to engage in upward social comparison (i.e., considering the "other" as superior) when assessing individuals with whom they have close relationships and are more likely to make upward (i.e., negative affect inducing) comparisons to distant friends and strangers (Wheeler \& Miyake, 1992), the higher number of non-close Facebook "friends" an individual has, the greater the tendency to compare upwardly. In some studies, perceived friend "closeness" correlates with regular online or offline interactions (Muscanell \& Guadango, 2012). Therefore, it is reasonable to hypothesize that Facebook users who visit the site often and have high numbers of non-close "friends", or friends with whom they have little personal interaction, will likely engage in more upward 
comparisons than those with fewer non-close friends or those who rarely use the site. Such comparisons should adversely affect self-perceptions, degrade selfworth, and result in lower reported levels of life satisfaction. This helps explain why users with more Facebook "friends" whom they do not know personally, or with whom they have very few or inconsistent interactions, are more likely to express less contentment with their own lives (Chou \& Edge, 2012).

Muise et al. (2009) support the expectations regarding possible negative responses to social comparisons via Facebook. They argue that there is "a significant association between time spent on Facebook and jealousy-related feelings and behaviors experienced on Facebook" (p. 443). The arousal of such emotions may be rooted in social-comparison jealousy that comes from a "desire for superiority on some dimension" (Salovey \& Rodin, 1984, p. 780). Because improving self-esteem is considered positive, degrading selfesteem and self-worth is the logical opposite. The connection between social comparison literature and observable potential Facebook behaviors results in the following hypotheses:

H1a: Facebook users with high numbers of friends they do not have frequent contact with report less life satisfaction than other users.

H1b: Facebook users who have more frequently contacted Facebook friends report greater life satisfaction than users who have less frequently contacted Facebook friends.

$\mathrm{H} 2$ : The more time users spend on Facebook, the lower their reported level of life satisfaction.

\subsection{Self-Construal and Gender Differences}

Existing scholarship also provides insight into possible expectations regarding sex and gender differences in Facebook use and its varying impacts. Social identification theories related to self-construal help to deepen our understanding of notable sex- and gender-based differences. The theory of self-construals helps us understand the differences in communication and social interactions (Tarr, Kim, \& Sharkey, 2005) and explains how individuals perceive themselves in relation to the social world around them (Cross \& Madson, 1997). The theory includes assessments of the contributions various communication strategies make toward an individual's self-perceptions (Tarr et al., 2005).

Similar to the cultural assertions regarding "individualism" and "collectivism" (Markus \& Kitayama, 1991), self-construal generally classifies individuals' selfperceptions into two opposing yet complementary categories: "independent self-construals" and "interdependent self-construals" (Kim \& Sharkey, 1995). People demonstrating independent self-construals perceive themselves as having a "bounded, unitary, stable self that is separate from the social context" (Tarr et al., 2005 , p. 500). Those with interdependent selfconstruals have a "flexible, variable self" rooted in their relationships with others (Sharkey \& Singelis, 1995, p. 920). The primary communication goals of an independent self-construal include standing out, being expressive and demonstrating his or her "unique internal characteristics or traits" (Tarr et al., 2005, p. 500). Conversely, individuals with interdependent self-construals unite perceptions of their own selfhood with those of others, focus on relationship maintenance and emphasize the "need to support others' face" (Tarr et al., 2005 , p. 500). That is, interdependent self-construals are developed in relation to others and the "other" is central to the identity of the interdependent person's understanding of self.

Cross and Madson (1997) utilize the concept of selfconstrual to elucidate gendered communication differences among members of what is classically considered the "individual-centric" culture of the United States. Their application of self-construal theories demonstrates gender differences within the culture, particularly regarding social participation. Cross and Madson (1997) find that men tend to be more independent self-construals while women generally have more interdependent self-construals. While not categorically exclusive, the dominant tendencies indicate that women are inclined to be more relationship-oriented, more indirect in their communication and focus more on the "other" than the self (Cross \& Madson, 1997) while men are more focused on self-development, selfpromotion, and self-expression. In studies of individual self-representations, women more often identify themselves and their ideal self in terms of relationship to others while men represent themselves via personal attributes and individual characteristics (Cross \& Madson, 1997). In terms of information processing, women tend to be more attentive to others, remember faces and demonstrate a more "other-centric" assessment of relational exchanges (Cross \& Madson, 1997). These differences provide insights into possible distinctions between males' and females' use of Facebook.

Applying the self-construal concept to Facebook, Muscanell and Guadagno (2012) demonstrate a gender distinction in motivations and usage. Their study indicates that females engage in more relationship maintenance via the social networking site while males use Facebook for practical purposes. They found that women engage more in specific relationship development activities such as posting messages, sending private messages, posting pictures, and sending friend requests than men. Men more frequently seek potential dates, play games and look for event information (Muscanell \& Guadango, 2012).

While the existing literature demonstrates that females and males visit Facebook at similar rates (Chou \& Edge, 2012; Hum, et al., 2011; Muscanell \& Guadagno, 
2012; Pempek et al., 2009; Quan-Haase \& Young, 2010; Sukes et al., 2012), women are more likely than their male counterparts to engage in behaviors such as reading the posts of others and looking at others' photos (Muscanell \& Guedagno, 2012; Smock et al., 2011; Tosun, 2012). Males were found to be more active in selfexpression activities than females (Smock et al., 2011). However, it remains to be seen if these potential dissimilarities result in a different level of reported satisfaction with their lives.

In line with the aforementioned literature, this study pursues the following research questions:

RQ1: What is the difference between male and female participants' Facebook use? Do they differ in time, frequency, number of friends, or Facebook functions used?

Combining the implications and assumptions of both social comparison and self-construal literature, the negative impact Facebook has on female users' perceived levels of happiness and life satisfaction makes sense. Because women are more inclined to seek relational information online, they tend to view more pictures and read more posts than their male counterparts. This means they are more likely to receive a bigger mix of forced and sought information. They would also be more likely to interact with information from non-close friends than their male counterparts, resulting in more upward, and therefore negative, social comparisons. Based on prior studies, it seems feasible that these types of comparisons would tend to initiate, or in some cases reinforce, negative selfconstruals. However, because most prior construal studies were conducted on offline interactions, it is important to explore the potential connection in an online environment. Thus, it leads to a second research question:

RQ2: How are Facebook activities related to users' life satisfaction? And do any relationships between Facebook use and life satisfaction differ across genders?

\section{Methodology}

This study combines the results of two online surveys investigating respondents' use of Facebook and their reported levels of happiness and life satisfaction. The surveys were administered five months apart to separate sets of undergraduate students at a large, private research-one university in the United States. The initial survey included responses from 428 students. Of those respondents, $75 \%$ were female, and $16 \%$ were international students (non-U.S. residents). Participants selfreported their race/ethnic backgrounds as $72 \%$ White, $18 \%$ Asian, $8 \%$ Hispanic, and 2\% Black. Participants in the second survey were smaller in number but similar in demographic characteristics. Of 239 respondents, $72 \%$ were female, $19 \%$ were international students, 74.8\% were White, $15.7 \%$ Asian, $6.5 \%$ Hispanic, $2.6 \%$ Black and $0.4 \%$ Native American.

The initial survey consists of three parts: media use and preference (general to all media and specific to Facebook), evaluations of life, and demographics. Measures of Facebook use include number of Facebook "checks" per day and estimated length of log-ins. Questions regarding Facebook features consist of prompts regarding number of "friends" respondents have on Facebook, how many of these friends they have previously met in person, and how many Facebook friends respondents contacted regularly. It also includes questions regarding which Facebook-based activities respondents spend the time engaged in (i.e., posting on others' walls, private messaging, chatting, posting photos, playing games, etc.). Results from the initial survey led to the development of the second research question (how are Facebook activities related to users' life assessments and how does this differ across sex/gender) and the addition of questionnaire elements to the second survey.

To explore the question of the impact of Facebook activities on respondents' life satisfaction, all of the previous measures for Facebook use were repeated and expanded in the second survey. The second prompt contains similar measures with additional and more detailed questions to help explicate interesting points gleaned from the first survey. The second survey includes both closed- and opened-ended questions to gauge the motivation for Facebook use. For example, one of the open-ended questions asks, "why do you use Facebook?" The closed-ended questions examine attitudes toward Facebook's various features using Likert-scaled response choices. Such questions include a five-point scale asking respondents to indicate their level of agreement or disagreement with comments such as "Facebook is good for meeting new people" and "I prefer reading others' posts on Facebook rather than posting myself." There are also questions measuring respondents' agreement (or disagreement) with various statements about Facebook and its perceived usage. These include comments such as "People use Facebook to brag about their lives," "I get jealous when I look at other people's Facebook pictures," and "I judge others based on how many Facebook friends they have." These questions provide measures of respondents' attitudes about Facebook and Facebook users.

The second survey includes more detailed measures of life satisfaction and happiness. Respondents were asked to rate statements related to their own life satisfaction and happiness. Survey participants were asked to rate their agreement with phrases such as, "My life is fulfilling," "My life is meaningful," "I have a good life," "I am a happy person," "I am happy with my life 
now," "Life is generally fair," and "Others have better lives than I do." The scale runs from 1 = "most agree" to $5=$ "least agree," which was recoded in data analysis to reflect that the higher the number, the more positive the participant's assessment. A factor analysis of these measures with the Varimax rotation method resulted in the creation of a single factor that helped determine respondent's perceptions of their overall "life satisfaction."

A variety of statistical analyses were conducted in this study to extricate information from the survey responses. These include factor analyses, ANOVAs, and a canonical correlational analysis. The participants' responses were examined as a whole and the female responses were also examined separately (although there was no significant variation in findings for the purely female data set compared to the full set). The results are summarized below.

\section{Findings}

Initial results from both surveys support existing claims regarding the use of Facebook by college-aged participants. Respondents to both surveys reported using Facebook daily in a series of numerous short-term sessions. The average respondent logs on 5-10 times per day, with sessions estimated to last between 5 and 10 minutes. Females have a higher tendency toward daily Facebook use $(r=.129, p<.01)$. However, the difference in overall estimated time spent on Facebook between male and female users is insignificant $(r=.043, p$ $=.376$ ). Thus, while female users report more regular use, overall exposure to Facebook content was similar between the sexes.

The reported tally of Facebook "friends" participants report having is remarkably consistent across the two surveys for this study and with prior research. In the first survey, over $90 \%$ of respondents indicated they had 500+ Facebook friends. ${ }^{1}$ In an upwardly adjusted measure, the second survey resulted in the mean, median and mode of number of Facebook friends converging at $650-700$ friends. Approximately $20 \%$ of respondents indicated that they have more than 1000 Facebook friends. When asked the number of Facebook friends with whom respondents had regular contact, in both surveys more than $75 \%$ indicated fewer than 20. This number was consistent across male and female respondents.

As explained earlier, a factor analysis of the seven measures of respondents' perceived happiness and contentment with their lives resulted in the creation of

\footnotetext{
${ }^{1}$ An initial flaw discovered via the exploratory study was underestimating the number of "friends" respondents would have. The scale initially stopped with a high of "500+" which proved to be far too low for any possibly meaningful discovery. The scale was adjusted upward for the second survey.
}

a single "life satisfaction" factor. This factor is found to be positively correlated with the number of all Facebook friends users have $(r=.136, \mathrm{n}=635, p<.001 ; r=$ $.216, \mathrm{n}=230, p=.001$ ), which contradicts with the hypothesis derived from social comparison theory. Therefore, $\mathrm{H} 1 \mathrm{a}$ is not supported.

The number of Facebook friends surveyed participants interact with regularly is also found to be positively correlated with their perceived life satisfaction in both surveys ( $r=.066, \mathrm{n}=634, p=.096 ; r=.039, \mathrm{n}=$ $230, p=.554$ ). But both correlation coefficients fail to reach significance level. Therefore, $\mathrm{H} 1 \mathrm{~b}$ is also not supported. Given that both overall Facebook friends and friends one interacts with regularly correlate positively with users' perceived life satisfaction researchers must reconsider the application of social comparison theory into social media. This interesting result warrants further exploration of interactions with various types of Facebook and real life friends.

Using the factor analysis-derived measure of "life satisfaction" revealed a strong correlation between respondents' life satisfaction and specific activities engaged in on Facebook. Frequency of engagement in interactive activities was strongly connected to lower perceptions of contentment. In both surveys, the more respondents reported using Facebook chat $(r=-.099, p$ $=.013 ; r=-.199, p=.003$ ) the less satisfied they reported being with their lives. User interactions with photos also significantly correlated with adverse perceptions of life satisfaction. Uploading photos $(r=-$ $.139, p<.001 ; r=-.178, p=.007)$, tagging photos $(r=-$ $.185, p<.001 ; r=-.282, p<.001)$ and looking at other's photos/videos $(r=-.145, p<.001 ; r=-.225, p=.001)$ all have a direct relationship with users' perceived contentment with their own lives. Similar results were found based on measures of time spent using each of these tools, although Facebook chat and uploading photos fell out of statistical significance. Time spent tagging photos $(r=-.123, p=.002 ; r=-.200, p=.002)$ and looking at others' photos/videos $(r=-.030, p=.010$; $r=-.258, p<.001$ ) were significantly correlated with negative reports of life satisfaction in both surveys.

The initial survey yielded mixed results regarding gender differences in Facebook use. Female participants tend to check out others' life presentations more frequently than their male counterparts $(r=.110, p$ $=.012$ ), although the time women spend on this Facebook function is not significantly longer than the men's time spent at these activities $(r=.039, p=.213)$. It is worth noting that women in the initial survey reported slightly lower levels of life satisfaction than their male counterparts-but the results are inconclusive because of low significance levels.

For the second survey, a one-way ANOVA was used to test differences in use of specific Facebook functions/activities based on the sex of the respondent. While males reported using wall posts, music and 
games more frequently than females, the most distinct differentiation came in engagement with photos/video. While "looking at others' photos" yielded a less than ideal .054 significance in difference $\left(F_{1}, 228=3.758\right)$, women's tendency to upload photos is significantly higher than their male counterparts $\left(F_{1,230}=10.315, p=\right.$ .002). Even more striking is women's tendency to "tag" photos more often than men $\left(F_{1,229}=16.924, p<.001\right){ }^{2}$

Not surprisingly, this study found that individuals who engaged with photos did so in multiple ways; several significant positive correlations were found within photo-related Facebook activities. Tagging photos is positively correlated at the $p<.01$ level with uploading photos, $r(236)=.679$; looking at others' photos, $r(234)$ $=.446$; and checking others' information, $r(235)=.325$. Uploading photos and looking at others' photos were similarly correlated with the same activities. Individuals who engaged with photos did so in numerous ways.

The second survey also shows that engaging with photos is significantly positively correlated with a wide variety of motivations for Facebook use. "Showing off pictures," which includes behaviors such as posting one's own photos and tagging photos, is positively correlated with the following reasons for using Facebook: "meeting others' expectations," $r(234)=.212, p<.01$; "comparing self to other," $r(233)=.353, p<.01$; "keeping track of others' lives," $r(234)=.318, p<.01$; "seeking information about a new acquaintance," $r(233)=.355, p$ < 01; "self-expression," $r(232)=.360, p<.01$; and "bragging about accomplishments," $r(232)=429, p<.01$. Many of these individual concepts relate to both creating social comparisons and expressing self-construals.

Survey responses to a five-point measure of agreement with the statement "I get jealous of others' Facebook pictures" were negatively skewed $(M=3.23, S D=$ 1.17), indicating that the respondents do not think Facebook pictures induce jealousy in them. However, results indicate that jealousy from Facebook pictures correlates with feeling less separated from others ( $r(235)$ $=.188, p<.01$ ) and judging others based on the number of Facebook friends they have $(r(234)=.357, p<$ .01). There is also a positive correlation between jealousy and the tendency to visit Facebook when feeling lonely $(r(235)=.356, p<.01)$. These findings suggest that further research is needed to explicate the connection between these actions and sentiments.

\footnotetext{
2 "Tagging" is a sharing behavior in which a person connects others to a photo by "naming" the person as associated with the photo. Individuals can "tag" their own photos or others' photos as a means of sharing.
}

There is an overall negative relationship between time spent on Facebook and users' assessment with their lives ( $r=-.154, p=.002)$. Therefore, hypothesis 2 is supported. This finding from the first survey led to an increased focus on the impact of specific Facebook activities on life satisfaction in the second survey. The results of the second survey demonstrate a vital difference the contribution-specific activities make to life assessments. More time allocated toward actively engaging with photos - tagging, uploading and looking at others' photos-is significantly negatively correlated with attitudes such as "Life is generally fair," "My life is meaningful," "I have a good life," and "I am a happy person." However, uploading photos and tagging photos are positively correlated with respondents' professed happiness with their current life (see Table 1). Negative relationships were also discovered with time spent engaging in other Facebook activities such as chatting, wall posts, and messaging. Time spent on wall posts is negatively correlated with attitudes of general fairness of life, $r(232)=-.156, p=.018$, and perceptions that "my life is meaningful," $r(233)=-.172, p=.008$, but is positively correlated with current happiness with life, $r(233)=.132, p=.043$. However, this mix of influences on time spent engaged in various activities leaves mixed results regarding RQ2 and will be explored more thoroughly in the discussion section.

In order to see the overall relationship between the 12 distinct Facebook activities and 7 Facebook users' life assessments, a canonical correlation analysis was conducted. This statistical procedure aims at extracting the maximum number of canonical variates explaining the two sets of variables. The result shows the canonical weights and canonical loadings, the latter of which indicate the correlation between the original variable and the canonical variate (see Table 2). The loadings from Facebook activities show that photo/video related activities contribute greatly to the canonical variate (loadings of "looking at other's photos/videos" and "tagging photos" are .840 and .567 , respectively). It seems that these windows to others' lives via the social network do have some influence over one's life assessments and happiness level. Only one canonical variate (time spent on Facebook) achieves a statistically significant canonical root-.201 $(p=.011)$; in other words, the time spent on Facebook's various activities can roughly explain $20 \%$ of Facebook users' life assessments. In another estimate, the overall correlation between the two sets of variables (Facebook activities and life assessments) is .449. 
Table 1. Correlation matrix between Facebook use and life assessments $(N=233)$.

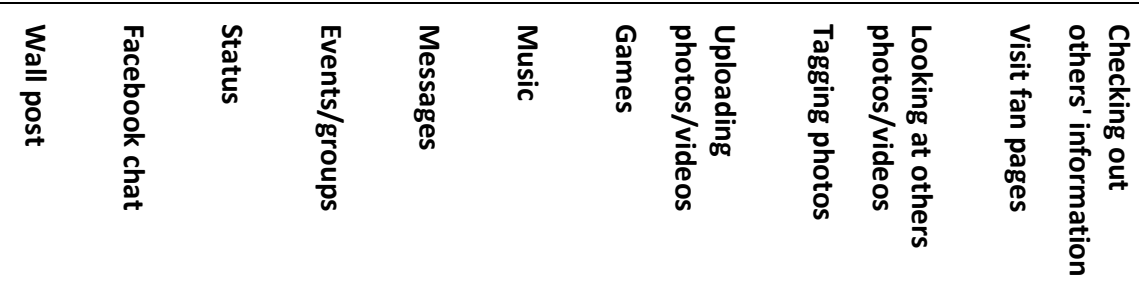

\begin{tabular}{|c|c|c|c|c|c|c|c|c|c|c|c|c|}
\hline \multicolumn{13}{|l|}{ Frequency of using } \\
\hline How happy are you with your life now? & .132 & .033 & .109 & .107 & .076 & .046 & -.028 & .137 & .141 & .021 & .064 & -.056 \\
\hline I have a good life. & -.115 & -.211 & .004 & -.248 & -.178 & .165 & .097 & -.075 & -.188 & -.145 & -.050 & -.032 \\
\hline Life is generally fair. & $\underline{-.156}$ & -.129 & -.061 & -.193 & -.165 & .041 & -.089 & -.169 & -.277 & -.098 & -.051 & .009 \\
\hline I am a happy person. & -.096 & -.181 & -.003 & -.231 & -.109 & .083 & -.039 & -.174 & -.278 & -.260 & .034 & -.047 \\
\hline My life is meaningful. & -.172 & $\underline{-.170}$ & -.095 & -.229 & -.077 & .012 & -.014 & -.173 & -.213 & -.251 & -.072 & -.079 \\
\hline Others have better lives than I do. & -.026 & -.010 & -.026 & -.019 & .010 & .037 & .023 & .001 & .042 & .002 & -.028 & .014 \\
\hline My life is fulfilling. & -.123 & -.177 & -.059 & -.216 & -.088 & .100 & .032 & -.114 & -.233 & -.183 & -.037 & -.070 \\
\hline I take joy in being social. & -.250 & -.262 & -.060 & -.313 & -.224 & .031 & .030 & -.257 & -.382 & -.314 & .054 & -.182 \\
\hline I am shy in face-to-face interactions. & -.083 & -.041 & -.037 & -.033 & -.082 & .193 & .267 & -.068 & -.121 & -.103 & .080 & .043 \\
\hline I am an outgoing person. & -.200 & -.173 & -.102 & -.247 & -.124 & .029 & -.014 & -.206 & -.315 & $=.212$ & .016 & -.069 \\
\hline \multicolumn{13}{|l|}{ Amount of time } \\
\hline How happy are you with your life now? & .011 & .052 & .072 & .110 & .112 & -.039 & -.059 & .079 & .084 & .168 & .003 & -.086 \\
\hline have a good life. & -.034 & -.164 & .061 & -.127 & -.104 & .187 & .079 & -.038 & -.136 & -.181 & .092 & .066 \\
\hline Life is generally fair. & -.031 & -.116 & -.015 & -.095 & $\underline{-.145}$ & .002 & -.047 & $\underline{-.151}$ & -.177 & -.056 & .011 & -.002 \\
\hline I am a happy person. & .052 & -.069 & .056 & -.111 & -.108 & .135 & .014 & -.162 & -.262 & -.291 & .094 & -.016 \\
\hline My life is meaningful. & .014 & -.133 & -.060 & $\underline{-.160}$ & -.080 & .079 & .021 & -.099 & -.174 & -.279 & -.032 & -.019 \\
\hline Others have better lives than I do. & .107 & .083 & -.067 & -.001 & -.020 & .108 & .121 & .064 & .020 & .076 & .089 & .154 \\
\hline My life is fulfilling. & -.004 & -.126 & .010 & $\underline{-.136}$ & -.082 & .138 & .066 & -.058 & -.120 & -.200 & .066 & -.001 \\
\hline I take joy in being social. & -.090 & -.126 & .035 & -.206 & -.102 & .128 & .088 & -.173 & -.301 & -.320 & .192 & -.061 \\
\hline I am shy in face-to-face interactions. & -.043 & .021 & .020 & -.035 & .051 & .194 & .249 & -.081 & -.104 & -.108 & .193 & .020 \\
\hline I am an outgoing person. & -.100 & -.065 & -.053 & -.192 & -.054 & .090 & .049 &.- .160 & -.237 & -.317 & .147 & -.028 \\
\hline
\end{tabular}

Note: *Presented in the table are Pearson coefficients, underline $p<.05$; bold $p<.01$.

Table 2. Canonical structure between the two sets of variables.

\begin{tabular}{|c|c|c|}
\hline & Canonical Weight & Canonical Loading \\
\hline \multicolumn{3}{|l|}{ Facebook Activity } \\
\hline Wall post & -.155 & -.022 \\
\hline Facebook chat & .099 & .263 \\
\hline Status & -.063 & -.001 \\
\hline Events/groups & .341 & .444 \\
\hline Messages & .093 & .218 \\
\hline Music & -.134 & -.106 \\
\hline Checking out others' Info & -.051 & .159 \\
\hline Uploading photos or/and videos & .197 & .433 \\
\hline Tagging photos & .155 & .567 \\
\hline Looking at other photos/videos & .731 & .840 \\
\hline Games & .116 & .039 \\
\hline Visit fan pages & .028 & .046 \\
\hline \multicolumn{3}{|l|}{ Life Assessment } \\
\hline I have a good life & -.041 & .562 \\
\hline Life is generally fair & -.062 & .269 \\
\hline I'm a happy person & .520 & .807 \\
\hline My life is meaningful & .680 & .824 \\
\hline Others have better lives than I do & .333 & .119 \\
\hline My life is fulfilling & -.244 & .599 \\
\hline I am happy with my life now & .311 & .535 \\
\hline
\end{tabular}


Measures of respondents' use of Facebook tools also yielded interesting results. The initial survey results indicated that "looking at others' photos" was the most frequently engaged in activity on Facebook (23\%) and was how users spent the highest proportion of their Facebook time (30\%). Of all respondents to the second survey, $49 \%$ indicate "looking at others' photos/videos" is an activity they "very frequently" engage in. When "frequent" and "very frequent" use responses were combined, $89 \%$ of respondents declared "looking at others' photos/videos" to be a highly used feature of the social network site. The next most frequently engaged in activities on Facebook were "messaging" (86.9\% selected frequent or very frequent combined) and "wall posts" $(83.5 \%$ frequent or very frequent combined). Conversely, game functions were used least frequently. Almost $70 \%$ of respondents report "never" using Facebook to play online games. The variety in the types of Facebook tools used, or not used, by respondents provides some insight into the ways these users interact with the information available via the medium and enhance our understanding of why there are such correlations between Facebook use and life satisfaction.

\section{Discussion}

This study examines the impact of Facebook use on college-aged individuals' life satisfaction. The discoveries across the two surveys do not support the first set of hypotheses about the relationship between the amount of Facebook friends and life satisfaction. There appears to be a need for continued exploration. The existing literature about social comparison may first appear relevant in the social media setting, but the relationships formed on social media and their impact on one's perceived life satisfaction may be based on other psychological concepts. The consistent correlation between the numbers of Facebook friends one has and his overall life satisfaction and the inconclusive finding of the impact of frequently interacted Facebook friends warrant further exploration. Perhaps a future study focusing specifically on the interactive differences based on friendship types may shed new light on this intriguing phenomenon.

Hypothesis 2 is fully supported, confirming a strong connection between the amount of time someone spends on Facebook and his or her perceived life satisfaction. This finding is consistent with existing research regarding Facebook use (Chou \& Edge, 2012; Kross et al., 2013; Muise et al., 2009; Ryan \& Xenos, 2011; Sukes et al., 2012). However, this study leaves the question open as to why such a negative correlation exists. Others have hypothesized that the connection may be due to a motivational disappointment (i.e., Facebook does not gratify the needs the user seeks to fulfill) (Smock et al., 2011) or that the asynchronous na- ture of the medium allows for the presentation of an edited or "best self" version of others' lives that no one's own life can live up to (Park, Lee, \& Kim, 2012; Tosun, 2012; Wilcox \& Laird, 2000). This study, although not positioned to make causal claims, hints at the ways in which users engage with Facebook content may influence the impact of the medium.

The second research question for this study asks how users' Facebook behaviors impact their life satisfaction. A clear general connection was found between the activities Facebook users engage in online and their overall life satisfaction. However, our data analysis does not provide a straightforward explanation about the social medium's impact on life satisfaction. The complex results regarding RQ2 warrant further discussion, as do the implications of this study for the listed research questions.

While previous literature suggests that increased time spent on Facebook should result in overall lower levels of happiness (Chou \& Edge, 2012) this more granular approach parses out the impact of particular Facebook activities and provides potential insights into why those activities alter perceived life satisfaction based on social comparison and self-construal literature. This study reveals a pattern of respondents feeling less satisfied with their lives the more they engage in interactive activities that include a mix of "forced" information (pictures and wall posts) and "sought" information (messages and chatting), understanding that all information is, to some degree, generally sought when using Facebook. Existing literature on social comparisons suggests that sought information should create downward comparisons and therefore create positive reflections of self (Kim \& Sharkey, 1995; Wheeler \& Miyake, 1992). However, that is not the case here. Perhaps the distinction between sought and forced information is less important in this situation than another shared characteristic of these types of activities. The more telling commonality here is that these activities all encourage specific consideration of others' information. More individualized Facebook activities (those not requiring interacting with others' personal information-e.g., music, games, personal status updates) showed no statistically significant correlation with life satisfaction assessments but activities such as tagging photos requires specific examination of others' pictures-participants must consider the content of someone's photo in order to engage in this activity. Perhaps it is the interaction with others' information and not the specific category of information that stimulates social comparisons and alters selfconstruals, therefore, influencing overall happiness and life satisfaction.

The impact of interacting with photos on Facebook provides some support for the idea that interactive social information seeking activities have a stronger negative impact than more solitary, self-focused activities 
do. Tagging photos implies an interest in relational development and maintenance because the point of tagging is creating a connection between the information and the person "tagged" and, through that, a connection to the "tagger." A person who tags must consider the content of the photo, determine the appropriate person(s) to tag based on social reasoning, and actually take the time to tag them. While not a highly technical process, it does require a high degree of intention on the part of the tagger. The interdependent construal characteristics exhibited by women (Cross \& Madson, 1997) may explain why female Facebook users have a higher tendency toward engaging in tagging activities. Also, interacting so purposefully with information about others is traditionally viewed as increasing the likelihood of creating social comparisons (Wheeler \& Miyake, 1992). It is not surprising, then, that female users tend to engage in these Facebook activities often and that this engagement corresponds with alterations in perceptions of one's satisfaction with her own life.

This study supports the idea that there are some gender-based differences in Facebook use and impacts. While the differences were not extensive, some were quite clear. Males and females engage in different Facebook activities and those activities impact their perceived levels of life satisfaction. Unfortunately, the disproportionate number of female respondents in each of the surveys for the present study may have masked further significant gender differences. Muscanell \& Guadagno (2012) had a disproportionately high concentration of males (65\%) in their study and had modestly different findings in male use patterns. For example, no respondent for either survey for this study cites "dating" as a reason or activity on Facebook, but it was a major factor in Muscanell and Guadagno's (2012) study. A separate assessment of the current data based solely on responses by females was consistent with the findings as stated here. A third iteration of the present survey should strive for a more balanced sample of males and females if more gender-based research questions are to be examined.

\section{Conclusion}

Facebook appears to be a rather promising medium for establishing and maintaining social connections (Chueng et al., 2011; Quan-Hass \& Young, 2010). However, if Facebook users seek happiness via this social connection, they are likely to be disappointed. Extensive use of online networking, for many, actually leads to increased dissatisfaction with their lives.

The findings of this study, supported by existing literature, show that Facebook may create sociocognitive tension for users. The strong motivation to acquire social information encourages Facebook use as a means of gratifying the need to attain social capital. The likely upward social comparisons resulting from the generally positive information available via Facebook may result in the skewed interpretations discovered by Chou and Edge (2012) and supported here.

From a theoretical perspective, this study demonstrates that while there are ties between existing interpersonal and self-perception based theories and mediated interpersonal and group networking, the theories are not necessarily directly applicable across platforms. The ideas of social comparison and selfconstruals logically connect with Facebook activities, but the offline findings do not directly or perfectly align with online behaviors or outcomes. This means that while the existing theories may act as guides, like other communication theories, they must be tested in the digital networking environment and adapted according to the contexts in which they are applied. Social comparison and self-construal theories may inform our understanding of online social networking, but require further exploration in mediated interpersonal settings.

Overall, this study provides interesting insights into the Facebook use patterns of college-aged population. The fact that Facebook use may engender an unanticipated, inverse impact is intriguing. Facebook users are driven to develop social connections to gratify the need to increase self-esteem by increasing social capital. However, the result of increased Facebook use, particularly interactions with photos and Facebook messaging, increases individuals' likelihood of engaging in social comparisons (including negative upward comparisons) and developing negative self-construals (particularly for females). These tendencies often serve to decrease a user's overall satisfaction with his or her life. The tension created by this dissonance between the social connectedness one seeks and the dissatisfaction one attains, however, does not seem to decrease the tendency to use the social networking system. While social comparison and self-construal research provide an initial, but only partial, explanation for this phenomenon, more research is necessary to fully understand both the causes of the dissatisfaction and users' responses to it.

\section{Acknowledgments}

The authors wish to thank all the student participants at Boston University.

\section{Conflict of Interests}

The authors declare no conflict of interests.

\section{References}

Antheunis, M. L., Valkenburg, P. M., \& Peter, J. (2010). Getting acquainted through social network sites: Testing a model of online uncertainty reduction and social attraction. Computers in Human Behavior, 26, 
100-109. doi:10.1016/j.chb.2009.07.005

Antoci, A., Sabatini, F., \& Sodini, M. (2012). See you on Facebook! A framework for analyzing the role of computer mediated interaction in the evolution of social capital. The Journal of Socio-Economics, 41, 541-547. doi:10.1016/j.soec.2012.04.024

Arundale, R. B. (2010). Constituting face in conversation: Face, facework, and interactional achievement. Journal of Pragmatics, 42, 2078-2105. doi:10.1016/j.pragma.2009.12.021

Attrill, A., \& Jalil, R. (2011). Revealing only the superficial me: Exploring categorical self-disclosure online. Computers in Human Behavior, 27, 1634-1642. doi:10.1016/j.chb.2011.02.001

Baek, K., Holton, A. Harp, D., \& Yaschur, C. (2011). The links that bind: Uncovering novel motivations for linking on Facebook. Computers in Human Behavior, 27, 2243-2248. doi:10.1016/j.chb.2011.07.003

Brewer, M. B., \& Weber, J. G. (1994). Self-evaluation effects of interpersonal versus intergroup social comparison. Journal of Personality and Social Psychology, 66, 268-275.

Bumgarner, B. A. (2007). You have been poked: Exploring uses and gratifications of Facebook among emerging adults. First Monday, 12. Retrieved from http://firstmonday.org/article/view/2026/1897

Cheung, C. M. K., Chiu, P., \& Lee, M. K. O. (2011). Online social networks: Why do students use Facebook? Computers and Human Behavior, 27, 13371343. doi:10.1016/j.chb.2010.07.028

Chou, H. G., \& Edge, N. (2012). "They are happier and having better lives than I am": The impact of Facebook on perceptions of others' lives. Cyber Psychology, Behavior and Social Networking, 15, 117-121. doi:10.1089/cyber.2011.0324

Cross, S. E., \& Madson, L. (1997). Models of the self: Self-construals and gender. Psychological Bulletin, 122, 5-37.

Ellison, N. B., Steinfield, C., \& Lampe, C. (2007). The benefits of Facebook "friends": Exploring the relationship between college students' use of online social networks and social capital. Journal of Computer-Mediated Communication, 12, 1143-1168.

Ellison, N. B., Steinfield, C., \& Lampe, C. (2011). Connection strategies: Social capital implications of Facebook-enabled communication practices. New Media Society, 13, 873-892. doi:10.1177/14614448 10385389

Engagement Analytics, Facebook, United States. (2012). Retrieved from www.socialbakers.com/face book-statistics/united-states

Ferlander, S. (2007). The importance of different forms of social capital for health. Acta Sociologica, 50, 115-128. doi:10.1177/0001699307077654

Fischer, P., Kastenmuller, A., Frey, D., \& Peus, C. (2009). Social comparison and information transmission in the work context. Journal of Applied Social Psychol- ogy, 39, 42-61.

Hum, N. J., Chamberlin, P. E., Hanbright, B., Portwood, A. C., Schat, A., \& Bevan, J. L. (2011). A picture is worth a thousand words: A content analysis of Facebook profile photographs. Computers and Human Behavior, 27, 1828-1833. doi:10.1016/j.chb.2011. 04.003

Hwang, Y. (2011). Is communication competence still good for interpersonal media? Mobile phone and instant messenger. Computers in Human Behavior, 27, 924-934. doi:10.1016/j.chb.2010.11.018

Kim, M. S., \& Sharkey, W.F. (1995). Independent and interdependent construals of the self: Explaining cultural patterns of interpersonal communication in multi-cultural organizational settings. Communication Quarterly, 43, 20-38.

Kross, E., Verduyn, P., Demiralp, E., Park, J., Lee, D. S., Lin, N., Shablack, H., Jonides, J., \& Ybarra, O. (2013). Facebook use predicts declines in subjective wellbeing in young adults. PLOS ONE, 8(8), e69841. doi:10.1371/journal.pone.0069841.

Markus, H. R., \& Kitayama, S. (1991). Culture and the self: Implications for cognition, emotion and motivation. Psychological Review, 98, 244-253.

Muise, A., Christofides, E., \& Desmarais, S. (2009). More information than you ever wanted: Does Facebook bring out the green-eyed monster of jealousy? Cyber Psychology \& Behavior, 12, 441-444. doi:10.1089/cpb.2008.0263

Muscanell, N. L., \& Guadagno, R. E. (2012). Make new friends or keep the old: Gender and personality differences in social networking use. Computers in Human Behavior, 28, 107-112. doi:10.1016/j.chb. 2011.08.016

Nadkarni, A., \& Hofmann, S.G., (2011). Why do people use Facebook? Personality and Individual Differences, 52, 243-249. doi:10.1016/j.paid.2011.11.007

Nosko, A., Wood, E., \& Molema, S. (2010). All about me: Disclosure in online social networking profiles: The case of FACEBOOK. Computers and Human Behavior, 26, 406-418. doi:10.1016/j.chb.2009.11.012

Park, N., Lee, S., \& Kim, J.H., (2012). Individuals' personal network characteristics and patterns of Facebook use: A social network approach. Computers in Human Behavior, 28, 1700-1707. doi:10.1016/ j.chb.2012.04.009

Pempek, T. A., Yermolayeva, Y. A., \& Calvert, S. L. (2009). College students' social networking experiences on Facebook. Journal of Applied Developmental Psychology, 30, 227-238. doi:10.1016/j.appdev. 2008.12.010

Quan-Haase, A., \& Young, A. L. (2010). Uses and gratifications of social media: A comparison of Facebook and instant messaging. Bulletin of Science Technology \& Society, 30, 350-361. doi:10.1177/02704676 10380009

Ryan, T., \& Xenos, S., (2011). Who uses Facebook? An 
investigation into the relationship between the Big Five, shyness, narcissism, loneliness, and Facebook usage. Computers in Human Behavior, 27, 16581664. doi:10.1016/j.chb.2011.02.004

Salovey, P., \& Rodin, J. (1984). Some antecedents and consequences of social-comparison jealousy. Journal of Personality and Social Psychology, 47, 780792.

Sharkey, W. F., \& Singelis, T. M. (1995). Embarrassability and self-construal: A theoretical integration. Personality and Individual Differences, 19, 919-926.

Sheldon, K. M., Abad, N. \& Hinsch, C. (2011). A twoprocess view of Facebook use and relatedness need-satisfaction: Disconnection drives use and connection rewards it. Journal of Personality and Social Psychology, 100, 766-775. doi:10.1037/ a0022407

Smock, A. D., Ellison, N. B., Lampe, C., \& Whon, D. Y. (2011). Facebook as a toolkit: A uses and gratification approach to unbundling feature use. Computers in Human Behavior, 27, 2322-2329. doi:10. 1016/j.chb.2011.07.011

Sukes, J. L., Williams, B., \& Wise, L. (2012). The effects of personality traits, self-esteem, loneliness, and narcissism on Facebook use among university students. Computers in Human Behavior, 28, 24142419. doi:10.1016/j.chb.2012.07.012

Tarr, N. D., Kim, M. S., \& Sharkey, W. F. (2005). The effects of self-construals and embarrassability on predicament response strategies. International Journal of Intercultural Relations, 29, 497-520. doi:10.1016/j.ijintrel.2005.07.002

Tosun, L. P. (2012). Motives for Facebook use and expressing "true self" on the Internet. Computers in Human Behavior, 28, 1510-1517. doi:10.1016/ j.chb.2012.03.018

Valkenburg, P. M., \& Peter, J. (2011). Online communication among adolescents: An intergrated model of its attractions, opportunities and risks. Journal of Adolescent Health, 48, 121-127. doi:10.1016/ j.jadohealth.2010.08.020

Vitak, J., Zube, P., Smock, A., Carr, C.T., Ellison, N., \& Lampe, C. (2011). It's complicated: Facebook users' political participation in the 2008 election. CyberPsychology, Behavior and Social Networking, 14, 107-114. doi:10.1089/cyber.2009.0226

Who Uses Facebook. (2012). Retrieved from www. arbitragemagazinf.com/topics/facebook-twitterinfographic

Wheeler, L., \& Miyake, K. (1992). Social comparison in everyday life. Journal of Personality and Social Psychology, 62, 760-773.

Wilcox, K., \& Laird, J.D. (2000). The impact of media images of super-slender women in women's selfesteem: Identification, social comparison and selfperception. Journal of Research in Personality, 34, 278-286. doi:10.1006/jrpe.1999.2281

\section{About the Authors}

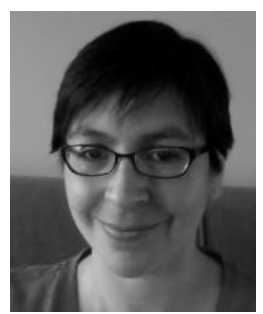

\section{Dr. Tammy R. Vigil}

Tammy Vigil is an assistant professor of communication studies at Boston University. Her research interests include political communication, rhetoric, and popular culture. Dr. Vigil coauthored The Third Agenda in U.S. Presidential Debates: Debate Watch and Citizen Reactions, 1996-2004 and edited a textbook titled Introduction to the World of Communication. Her book Connecting with Constituents: Identification Building and Blocking in Contemporary National Convention Addresses examines national convention rhetoric. Dr. Vigil is a past winner of the National Communication Association's Wrange-Baskerville award.

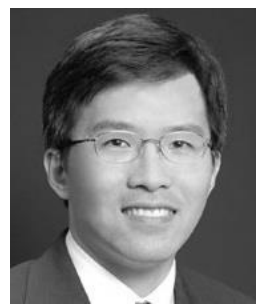

\section{Dr. H. Denis Wu}

$\mathrm{H}$. Denis $\mathrm{Wu}$ is an associate professor of communication at Boston University. His research areas are in international communication, new media, and political communication. His works have been published in esteemed academic journals such as Journal of Communication and Journalism \& Mass Communication Quarterly. His most recent book, Image and Emotion in Voter Decisions: The Affect Agenda (with Renita Coleman), was published by Lexington Books in 2015. Currently, he serves as an associate editor of Mass Communication \& Society. 